the "inactive" conformation, described by Fresco and others.

\section{How Red Cells Lose Nuclei}

\section{from our Correspondent in Celi Biology}

At long last some convincing electron micrographs have been obtained of nuclear expulsion during the final stages of erythrocyte maturation in mammals (Skutelsky and Danon, J. Cell Biol., 33, 625; 1967). Mature erythrocytes in circulating mammalian blood have no nuclei. The developing immature erythroblasts are nucleate cells. Two rival theories were proposed to explain the loss of the nucleus at late stages of maturation. One, primarily based on observations of primitive erythrocytic cell lines and some pathological conditions, was that the nucleus breaks down in situ, by karyolysis. The other, supported by Bessis and Bricka's microcinematography (1952), was that the nucleus is normally expelled from the cell. The supporters of the karyolytic theory objected that free nuclei are rarely scen in normal erythropoietic tissues.

Several electron microscopic studies failed to yield conclusive evidence for expulsion. Authors have often made vague statements that evidence for nuclear expulsion and/or karyolysis has been found, but have failed to include the vital micrographs in their articles. Part of the difficulty apparently arises from lack of suitable tissue to study. In normal erythropoietic tissue the erythroblasts are scattered among other cell types, which means that the chance of identifying extruded nuclei in thin sections is remote. Moreover, as the work of Bessis and Bricka shows, expulsion is rapid so that it is difficult to catch a nucleus halfway out.

Skutelsky and Danon have overcome these problems by inducing pure clones of erythropoietic cells in mice spleen by injecting syngeneic bone marrow into X-ray irradiated mice. In such clones cells at all the stages of maturation occur close together, so that the final stages involving loss of nuclei can readily be examined. As the micrographs show, nuclear expulsion does occur and the process closely resembles cell cleavage following mitosis. Skutelsky and Danon have found no evidence at all of karyolysis in the 20 clones examined.

Nearly mature erythroblasts develop cytoplasmic processes resembling pseudopods and the nucleus moves into one of these, often being deformed as it passes through the narrow neck. Within a process, the nucleus rounds up again and is surrounded by a very narrow rim of cytoplasm. The plasma membrane begins to constrict about the neck of the process containing the nucleus, leaving it attached to the main body of the cell by a very narrow bridge of cytoplasm. Mitochondria aggregate in the main cell body in the region of the constriction, and presumably provide energy for any contractile process involved in constriction and for the synthesis of new plasma membrane. Vacuoles then appear in the bridge region and effect the complete separation of the nucleus from the young reticulocyte. The nuclei released are soon engulfed by macrophage, so that free nuclei arc rarely seen in erythropoietic tissues.

Three questions are raised by these observations. First, what is it that triggers the whole process and brings about nuclear expulsion rather than a cell division? Secondly, what are the properties of the plasma membrane surrounding the expelled nuclous that make it readily recognizable by tho macrophage ?
Thirdly, can Skutelsky and Danon's obscrvations be generalized to definitive cell line erythroblasts in all mammalian erythropoietic tissue? The answer to the last question is probably affirmative, for it is most unlikely that the method used to induce pure clones of erythroblasts would completely alter the way in which the cells lose their nuclei, and there was no indication of karyolysis.

\section{Cancer Research}

\section{from a Correspondent}

The forty-fourth annual report of the British Empire Cancer Campaign, published this week, includes as in previous years reports of work in a wide variety of disciplines and, even though in 1966 the Campaign

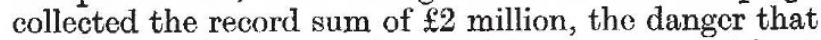
the money will be spread too thinly over a broad front of attack is unlikely to recede.

Some of the more basic projects described in the report for 1966 are concerned with the problems of growth regulation and control. According to P. N. Campbell and E. Lowe (Courtauld Institute), the higher rate of synthesis of protein in regenerating liver is not related to the availability of messenger RNA nor to any factor associated with the polysomes, but rather to the presence of an inhibitor in the membrane of the endoplasmic reticulum. Professor J. A. V. Butler and his colleagues at the Institute of Cancer Research, on the other hand, are concentrating on the physical and chemical properties of histones because these appear to be involved in the switching on and off of genetic information. G. V. Sherbert, at the same institute, reports that the histone fraction from calf thymus produces apparently specific malformations in early chick embryos. From a study of the control of the renewal of cells in mouse lung, Simnett and Heppleston (Newcastle upon Tyne) conclude that cell division in alveolar tissue is probably regulated by a mitotic inhibitor which persists in tissuc culture preparations of lung. The higher mitotic index seen in cultures of newborn lung tissue is associated with the absence of this inhibitor, though systemic stimulatory factors, possibly hormones, may also be involved. Several previously reported experiments have suggested that thymectomy enhances response to chemical carcinogens. Against this background, it is surprising to find the report by A. Flaks (Leeds) that the injection of neonatal thymus tissue into mice increased their response to a carcinogen injected during the neonatal period. But in its time the thymus, like man himself, plays many parts, and it is at present difficult to see the pattern of their interrelationships. According to Koller and his colleagues (Institute of Cancer Research) although thymic cells may restore immunc competence after thymectomy, they are not themselves capable of elaborating antibody; but attempts to restore immune competence in thymectomized mice by extracts of calf thymus have so far yielded negative results. A. Glucksmann and C. P. Cherry (Strangeways Laboratorics, Cambridge) have made a study of the somewhat neglected epithelial cell components of the thymus. Oestrogens promote the proliferation and secretory activity of these cells and testosterones elicit the opposite response. It is suggested that these effects may be related to the differences in response to chemical carcinogens of the salivary glands and of the genital 